\title{
RECENT ADVANCES IN LOCAL ANESTHESIA - OVERVIEW
}

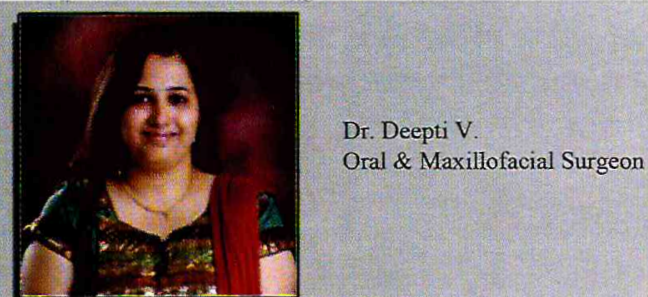

ABSTRACT

The local anesthetic drugs presently available and used in dentistry represent the safest and most effective drugs in all of medicine for the prevention and management of pain. One drawback associated with intraoral local anesthesia is patients' fear of injections and the perception that these are painful. Recent advances have resulted in the use of computer-controlled local anesthetic delivery vehicles to regulate the delivery and rate of flow of local anesthetics at the injection site, lessening potential discomfort associated with injections. New injection techniques that provide reliable anesthesia, depending on the technique and area of anesthesia necessary have been discussed.

KEY WORDS : Local Anesthesia, Fear Of Injections, Recent Advances, Computer-Controlled Technique.

\section{INTRODUCTION:}

Dental treatment has long been associated, in the mind of the patient, with pain. Indeed, fear of pain is one of the most significant factors deterring adults from receiving non-emergency dental care, even more so than the monetary cost of treatment.

The dental profession can take pride in its role in leading the development of the art and science of anesthesia.

Dr. Horace Wells, a Connecticut dentist, became the first person to use anesthesia for therapeutic reasons when he received $100 \%$ nitrous oxide (N2O) in December 1844 , prior to the extraction of a molar. ${ }^{1 t}$

\section{LOCAL ANESTHETICS:}

Carl Koller (1857-1944), an Austrian ophthalmologist, demonstrated the effect of cocaine as a local anesthetic for eye surgery in 1884 instilling drops of cocaine onto the surface of the eye, providing topical anesthesia. For the first time, a patient was able to undergo a surgical procedure awake and without pain.
William Halstead (1852-1922), administered an injection of cocaine (with epinephrine) via inferior alveolar nerve block for the removal of a neuroma.

\section{DESIRABLE ANESTHETIC PROPERTIES:}

A number of properties are desirable for local anesthetic agents and techniques. Efficacy, safety and biocompatibility are requirements. Other properties that are desired for an ideal agent and technique include a rapid onset, adequate duration and profundity of anesthesia, anesthesia of the targeted tissues and area only, rapid reversal, a lack of side effects and contraindications, a painless anesthetic delivery that is also unobtrusive, and a technique that is easy and has no, or a minimal, learning curve.

\section{RECENT ADVANCED LOCAL ANESTHETICS:}

Topically Applied Local Anesthetics

Topically applied anesthetics provide a degree of anesthesia to nonkeratinized tissue (e.g., oral mucous membrane) to a depth of 2 to $3 \mathrm{mms}$. This permits the initial penetration of mucous membrane to be accomplished painlessly. Application of a small amount of topical anesthetic to the injection site prior to needle penetration is an integral step in the delivery of atraumatic injections 2 .

Topical anesthetics are used in concentrations that are higher than those used when the drug is injected. For example, lidocaine injectable is a $2 \%$ solution, while when used topically as a gel or ointment it is commonly $5 \%$. Benzocaine, the most commonly used topical anesthetic, is used in a $20 \%$ concentration (gel, ointment, spray).

Oraqix, a recently introduced locally applied anesthetic gel, is a eutectic mixture of prilocaine and lidocaine, each in a $2.5 \%$ concentration.

Tricaine Blue, Tridocaine, TrioCaine and Profound topical are compounded topical anesthetics containing benzocaine, lidocaine and tetracaine. 
TRANSCUTANEOUS ELECTRICAL NERVE

STIMULATION (TENS) (THE 1980S):

In the 1980s, the medical technique transcutaneous electrical nerve stimulation (TENS) was modified for use in dentistry as a means of obviating the need for an injection of $L A$ for pain control - in other words, offering pain control without using needles. With electrodes applied intraorally and the current delivered at a higher frequency, pain control was achieved. Routine dental procedures,restorations, root planing and curettage were accomplished painlessly in a significant percentage of patients who used TENS. The dental application of TENS was renamed electronic dental anesthesia (EDA)3-5. When EDA worked, it worked well. Unfortunately, it turned out that EDA did not produce a consistently reliable level of pain control.

VIBRAJECT: The Vibraject utilizes vibration to reduce the sensation of pain during injections. This device utilizes a battery-powered attachment that is placed over a regular dental syringe and provides a series of fine vibrations to the needle tip during introduction of the local anesthetic. The Vibraject has been found to reduce pain during local anesthesia procedures and is not restricted to one injection technique. COMPUTER-CONTROLLED LOCAL ANESTHETIC DELIVERY SYSTEMS (THE 1990S-PRESENT):

In the mid-1990s, work began on the development of local anesthetic delivery systems that incorporated computer technology to control the rate of flow of the anesthetic solution through the needle. This concept is now called computer-controlled local anesthetic delivery (CCLAD)6. The first of these CCLAD devices, the Wand ${ }^{T M}$ (Milestone Scientific, Inc., Livingston, N.J.), was introduced in 1997. The system enabled a dentist or hygienist

to accurately manipulate needle placement with fingertip accuracy and deliver the LA with a foot-activated control. The lightweight handpiece is held in a pen-like grasp that provides the user with greater tactile sensation and control compared to a traditional syringe. The available flow rates of LA delivery are controlled by a computer and thus remain consistent from one injection to the next.

Several CCLAD systems are available, including the Wand/CompuDent ${ }^{\mathrm{Tm}}$ system, Comfort Control Syringe $^{\mathrm{TM}}$, QuickSleeper ${ }^{\mathrm{TM}}$ and Anaeject ${ }^{\mathrm{TM}}$. Both the Comfort Control Syringe and the Anaeject regulate the speed of injection, starting slowly and accelerating the speed of injection to minimize pain.
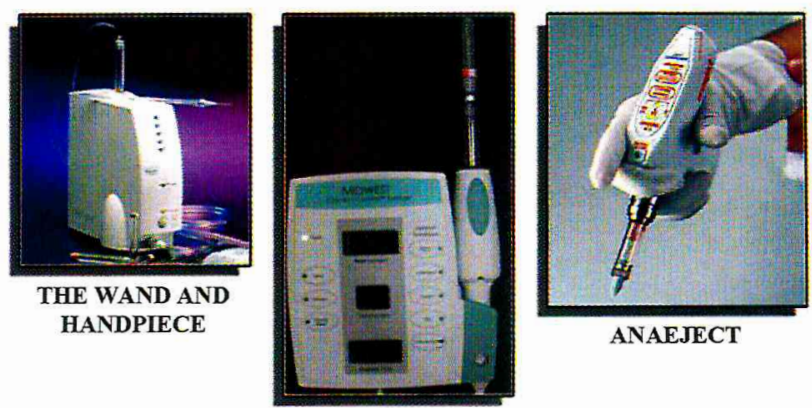

COMFORT CONTROL SYRINGE

P-ASA The palatal approach-anterior superior alveolar nerve block provides pulpal anesthesia to the six anterior teeth - canine to canine bilaterally as well as the palatal and labial gingiva and mucoperiosteum and bone overlying these teeth. As noted with the AMSA, there is no collateral anesthesia extraorally.

New Injection Techniques

Two new injection techniques, the AMSA7,8 and PASA9-13, have been described since the development of CCLAD.

AMSA The anterior middle superior alveolar nerve block provides pulpal anesthesia to the maxillary incisors, canines and premolars on the side of injection. Soft tissue anesthesia is achieved for the entire hard palate on both that side and the intraoral mucosa of the five anesthetized teeth. Significantly, no extraoral anesthesia develops with the AMSA, a benefit to both the patient (functionally and esthetically) and the doctor during cosmetic procedures (no drooping of the upper lip).

INTRAOSSEOUS ANESTHESIA:Intraosseous anesthesia involves the placement of local anesthetic directly into the cancellous bone spaces adjacent to the tooth or teeth that require anesthesia. This technique offers rapid onset of pulpal anesthesia.

Methods used have included the use of two-step and one-step techniques. Using a two-step technique, a bur is first used to penetrate the bone using a slow speed handpiece, after which the local anesthetic is placed.

A one-step technique (IntraFlow ${ }^{\mathrm{TM}}$ Anesthesia Delivery System) uses a slow-speed hand piece with a needle (perforator) and transfuser, resulting in penetration of the bone and immediate flow of anesthetic without a separate step. This technique uses a foot pedal to regulate flow.

SINGLE-TOOTH ANESTHESIA:In 2006, the manufacturers of the original CCLAD, the Wand,introduced a new device, Single Tooth Anesthesia (STA ${ }^{\mathrm{TM}}$ ).STA incorporates dynamic pressure-sensing (DPS) 
technology that provides a constant monitoring of the exit pressure of the local anesthetic solution in real time during all phases of the drug's administration.

STA utilizes an adaptation of DPS to dentistry as a means of overcoming the problems associated with PDL injection, and simplifies AMSA and P-ASA injections. The system can be utilized for all traditional in traoral injection techniques. STA includes a training mode that verbally explains how to use the device, and multi-cartidge and auto-cartridge retraction features.

\section{OTHER S}

Neosaxitoxin, a new local anesthetic derived from algae, had significantly less postoperative pain and recovered about two days sooner than those given the commonly used local anesthetic bupivacaine 15 . Neosaxitoxin (neoSTX) provides local anesthesia for more than 24 hours. It is a site 1 sodium-channel blocker, part of a larger class of emerging anesthetics based on molecules derived from aquatic organisms.

Local anesthesia delivered through an inhalable nasal spray quickly travels down one of the face's primary nerves to the mouth, which could be more effective than injecting it into the gums with a needle16.

SUMMARY: Local anesthesia forms the backbone of pain control techniques in dentistry, and local anesthetics are the safest and most effective drugs in all of medicine for the prevention and management of pain. However, the administration of these drugs is the most frightening and uncomfortable part of the dental appointment for most patients. The needle is the most fear inducing part of the armamentarium for the delivery of LAs.

Over the years, many futile attempts have been made to provide clinically adequate pain control without the need for injection of drugs. Recent developments in CCLAD systems have made the delivery of local anesthesia to patients significantly more comfortable and, with the PDL, AMSA and P-ASA injections, considerably more successful. The ability to deliver painless injections and a desirable level and duration of anesthesia results in reduced patient fear, reduced patient stress (and therefore reduced stress for the clinician) and can aid patient compliance with current and future dental treatment.

Refrences:

1. Stanley F. Malamed, DDS Anesthetic Agents and Computer-Controlled Local Anesthetic Delivery (CCLAD) in Dentistry A Peer-Reviewed
Publication: www.ineedce.com

2. Malamed SF. Basic Injection Technique, in Malamed SF. Handbook of Local Anesthesia. 6th edition, CV Mosby, St. Louis 2004.

3. 14. Meechan JG, Gowans AL, Wellbury RR. The use ofa patient-controlled transcutaneous electrical nerve stimulation (TENS) to decrease the discomfort of regional anaesthesia in dentistry. A randomized controlled clinical trial. J Dent. 1998;26(5):417-20.

4. Hochman R. Neurotransmitter modulator (TENS) for control of dental operative pain. J Am Dent Assoc. 1988;116:208-12.

5. 16. Malamed SF, Quinn CL, Torgersen RT, Thompson W. Electronic dental anesthesia for restorative dentistry. Anesth Prog. 1989;36:195-7.6.Proceedings of the 1st Annual Computer-Controlled Local Anesthesia Delivery (C-CLAD) System meeting.Introductory remarks. New Orleans, La., Feb 2008.

7. Friedman MJ, Hochman MN. The AMSA injection: a new concept for local anesthesia of maxillary teeth using a computer-controlled injection system. Quintess Int. 1998;29(5):297-303.

8. 27. Malamed SF. Techniques of Maxillary Anesthesia, in Malamed SF. Handbook of Local Anesthesia. 6th edition, CV Mosby, St. Louis 2004, p. 213-217.

9. 28. Malamed SF. Techniques of Maxillary Anesthesia, in Malamed SF. Handbook of Local Anesthesia. 6th edition,CV Mosby, St. Louis 2004, p. 217-220.

10. 29. Friedman MJ, Hochman MN. P-ASA block injection: a new palatal technique to anesthetize maxillary anterior teeth. J Esthet Dent. 1999;11(2):63-71.

11. 30. Friedman MJ, Hochman MN. Using AMSA and P-ASA nerve blocks for esthetic restorative dentistry. Gen Dent. 2001;49(5):506-11.

12. Ram D, Kassirer J. Assessment of a palatal approachanterior superior alveolar (P-ASA) nerve block with the Wand in paediatric dental patients. Int J Paediatr Dent. 2006;16(5):348-51.

13. Friedman MJ, Hochman MN. P-ASA block injection: a new palatal technique to anesthetize maxillary anterior teeth. J Esthet Dent. 1999;11(2):63-71.

14. Malamed SF. The periodontal ligament (PDL) injection: an alternative to inferior alveolar nerve block Oral Surg. 1982;53:117-21

15. Clay Dillow Posted Inhalable Local Anesthesic Could Replace Painful Needles at the Dentist

16. Inhalable Local Anesthesia Could Render Tha Needle Obsolete

17. Ropivacaine - a new local anesthetic. 
CODS Alumni and College day celebrations 19 th and 20 th of February 2011
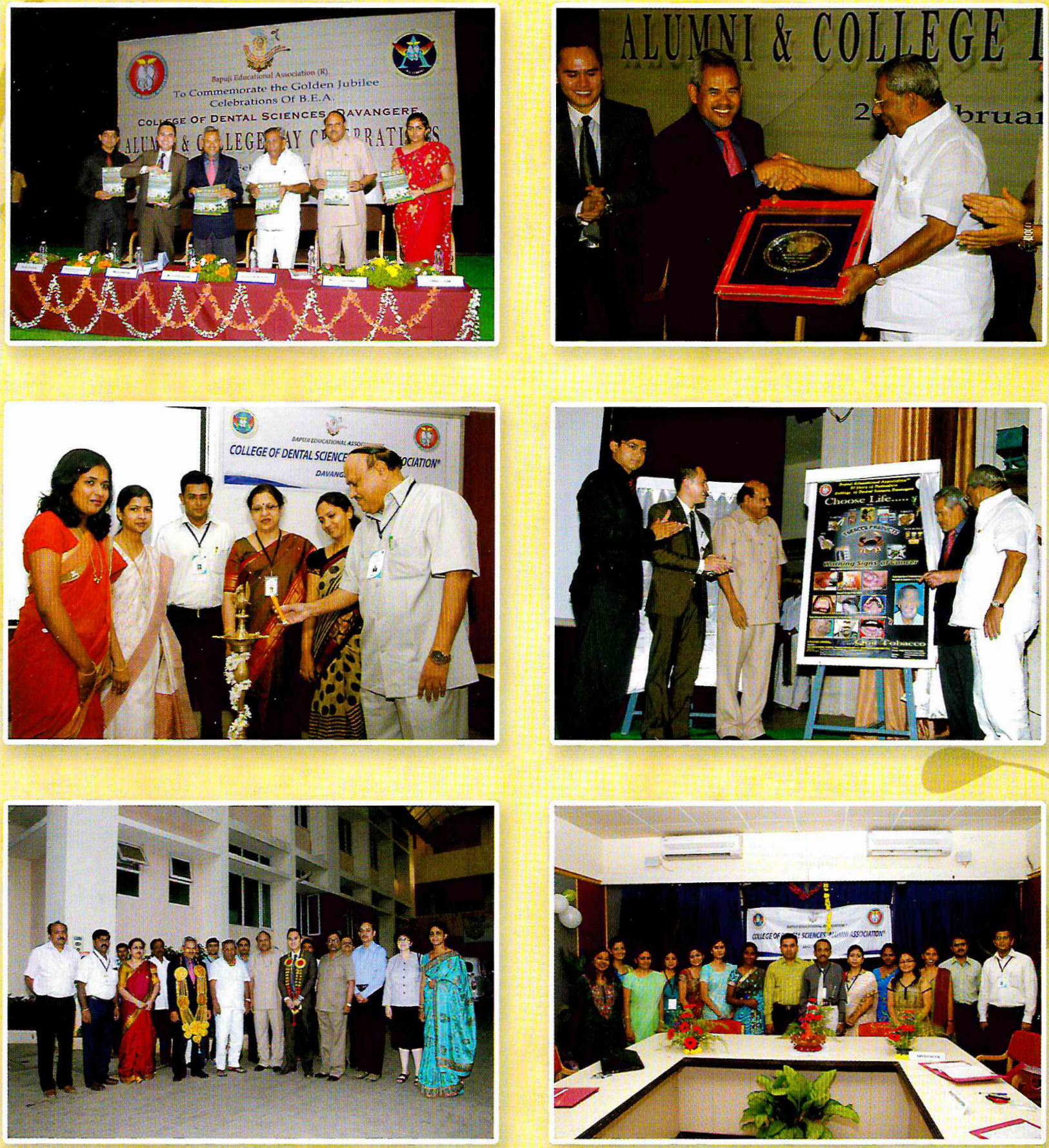

Chief Guest:

Sri Anuar Bin Kasman

Consul General of Malaysia (South India)

Guest of Honor:

Sri Khairudin Bin Haji Abduliah

Consul Education and Training (Malaysia)

Sri A S. Veeranna

Chairman, MBA College, Governing council member BEA

Dr. Shamanur Shivashankarappa MLA Chairman, College of Dental Sciences Hon. Secretary. Bapuji Educational Association Presided over the function 